\title{
Widely tunable Raman ring laser using highly nonlinear fiber.
}

\begin{abstract}
An all-optical widely tunable Raman fiber laser has been realized by incorporating a highly nonlinear fiber in a ring cavity. By feedback a portion of Raman Stokes wave back into the highly nonlinear gain medium, a Raman fiber laser is generated. We found that the lasing wavelength of Raman fiber laser can be tuned from 1537 to $1568 \mathrm{~nm}$ with peak power fluctuation within $1 \mathrm{~dB}$, giving a total wavelength tunability of $31 \mathrm{~nm}$. The optical signal-tonoise ratio is found to be wavelength dependent, and the highest optical signal-to-noise ratio of about $59 \mathrm{~dB}$ is recorded. The lasing threshold of the Raman fiber laser with this configuration is found to be as low as $300 \mathrm{~mW}$
\end{abstract}

Keyword: Raman fiber lasers; Fiber lasers; Ring lasers; Fibers. 